\title{
Reconstruction of the Sphenoid Wing in A Patient with Sphenoid Wing Dysplasia in NF1 - A Case Report
}

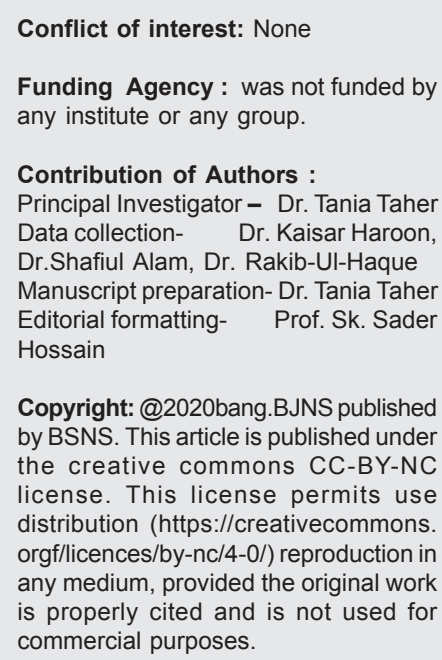

Copyright: @2020bang.BJNS published by BSNS. This article is published under the creative commons CC-BY-NC license. This license permits use distribution (https://creativecommons. orgf/licences/by-nc/4-0/) reproduction in any medium, provided the original work is properly cited and is not used for commercial purposes.

Taher $\mathrm{T}^{1}$, Haroon $\mathrm{K}^{2}$, Alam $\mathrm{S}^{3}$, Haque $\mathrm{RU}^{4}$, Hossain $\mathrm{S}^{5}$
Received: 12 April, 2019 Accept: 7 July, 2019

\begin{abstract}
:
Spheno-orbital dysplasia is almost pathognomic for Neurofibromatosis type 1. There is proptosis and deviation of the eye ball medially. We present a case of a young girl with neurofibromatosis type 1, who had pulsating exophthalmos of the right eye with diplopia and severe dysplasia of the sphenoid boneand consecutive herniation of the right temporal lobe. She presented with pulsatile proptosis and deviation of the right eye ball to the right. Her x-ray skull showed widening of the orbit. Her MRI scan showed an arachnoid cyst behind her right eye ball. We had operated on her and reconstructed the right sphenoid greater wing with titanium mesh and duroplasty with G-patch. This had improved her proptosis and deviation of the eye ball.
\end{abstract}

Key Words: Spheno-orbital dysplasia, neurofibromatosis type1, exophthalmos, sphenoid bone reconstruction,duroplasty; pulsatile proptosis.

Abbreviations: NF-Neurofibromatosis. CSF- cerebrospinal fluid

Bang. J Neurosurgery 2020; 9(2): 156-161
Neurofibromatosis type 1 (NF1) is an autosomal dominant inherited disease showing a plethora of signs and symptoms. Diagnostic criteria of NF1 include sphenoid wing dysplasia as a defining feature. This skeletal abnormality can be associated with an orbital plexiform neurofibroma or develops without any apparent associated pathology. Protrusion of the temporal lobe into the orbit via this defect can cause pulsating exophthalmos, leading to an unsightly appearance. Associated ophthalmologic pathologies in NF1 are well known and may also be present in the case of sphenoid wing dysplasia ${ }^{1}$.

Sphenoid wing dysplasia, perhaps more appropriately called aplasia, is a rare condition that is considered pathopneumonic for neurofibromatosis type 1 (NF1) ${ }^{2}$.

Sphenoid wing dysplasia is relatively rare in the general population and over $50 \%$ of the cases are associated with neurofibromatosis type 1 (NF1), a neurocutaneous disorder with an incidence of 1:3000. Sphenoid wing dysplasia occurs in $3-7 \%$ of patients with neurofibromatosis type $1^{3}$.

1. Dr. Tania Taher, Assistant Professor HFRC Medical College Hospital,

2. Dr. Kaisar Haroon, Assistant professor, Department of Neurosurgery, NINSH

3. Dr. ShafiulAlam, Associate Professor, Department of Neurosurgery, NINSH

4. Dr. Rakib-Ul-Haque, Medical Officer, Department of Neurosurgery, NINSH

5. Prof. Sk. Sader Hossain, Professor and Head, Department of Neurosurgery, NINSH

Address of Correspondence: Dr. Kaisar Haroon, Assistant Professor, Department of Neurosurgery, NINSH. Email:kaisar298@gmail.com, Phone +01711196577, ORCID: 0000-0002-3065-7877 
Sphenoid wing dysplasia may be progressive and may lead to disruption of the orbit, pulsating exophthalmos, and brain herniation into the orbit ${ }^{3}$.

Sphenoid wing dysplasia is estimated to affect up to $11 \%$ of persons with NF1 and has traditionally been thought to arise secondary to an adjacent soft tissue abnormality (e.g., plexiform neurofibroma). Another hypothesized explanation for the finding is a defective bone cell-autonomous program. Two lines of evidence support this second possibility. First, NF1 sphenoid wing lesions are significantly more likely to be associated with other dysplasias of the tibia and vertebrae and, second, the formation of the sphenoid bone arises from endochondral bone formation, which is known to be abnormal in NF14.

The characteristic skeletal abnormality in NF is the absence of the greater wing of the sphenoid, which may be partial or complete, allowing communication between the middle cranial fossa and the orbit. This defect results from widening of the superior orbital fissure and loss of the adjacent bone of the greater and lesser sphenoid wing ${ }^{5}$.

The effect on the sphenoid bone is progressive; normal becomes dysplastic, dysplastic becomes thin, thin becomes a gross defect, and gross defects increase in $\operatorname{size}^{6}$.

A competent frontal fossa floor is important in isolating the intracranial space from the contaminated paranasal sinuses and in preventing prolonged CSF leakage ${ }^{7}$.

Reconstruction of the orbit for this condition was first performed in 1927 by Dandy. Since that time, bone grafts have been the mainstay of repair. Due to problems with bone resorption and recurrence, titanium plates in conjunction with bone grafts have recently been reported ${ }^{2}$.

\section{Case report:}

A 16 year old female presented to us with pulsatile proptosis of the right eye and double vision. She stated that her eyes were normal during birth. But they started to deviate medially and inferiorly as she started to grow up. Now the right eye has come forward and is totally deviated medially and inferiorly. She noticed there is pulsation in the right eye, which is more during crying, coughing and sneezing. During sleep the pulsation would almost disappear. She was having double vision problem for a long time. On examination, she was averagely built girl. Her right eye had deviated medially and inferiorly and there was proptosis of the right eye. On neurological examination, there was no deficit. On local examination her right orbit was larger and the superior and inferior rim was absent. There was pulsation of the right eyeball without any bruit. The lateral canthus was lower on the right side with presence of ptosis. There was a tiny bony defect at the right side of her head. There were multiple Cafeau-lait spots in all over her body.

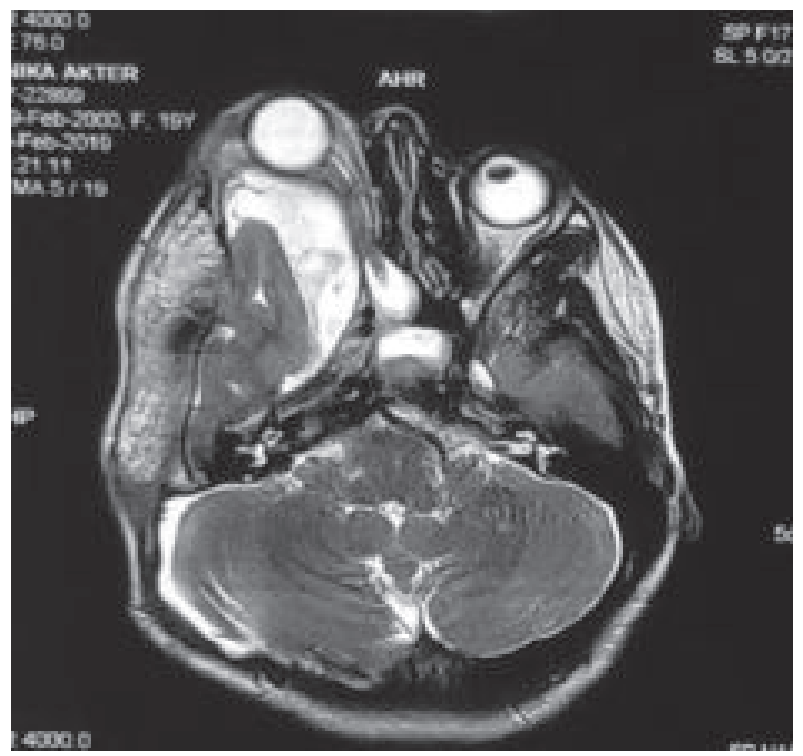

Fig.-1: T2WI MRI showing Rt. Orbital proptosis and eight sided retro-orbital arachnoid cyst

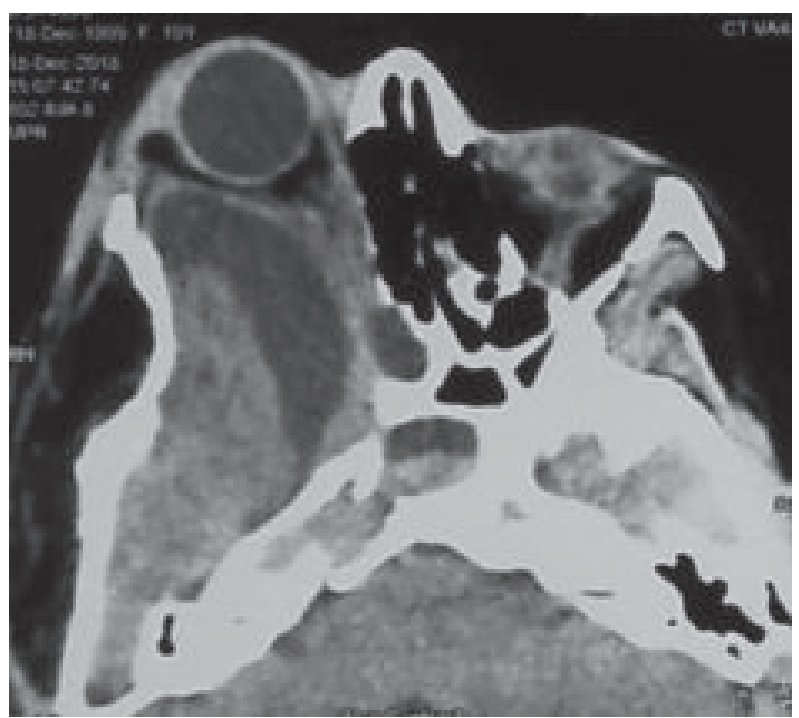

Fig.-2: Axial CT scan of brain showing Right orbital proptosis, Retro-orbital arachnoid cyst and deficient greater wing of sphenoid bone. 
Her CT scan (fig2) of the head showed the posterior superior and lateral orbital wall was absent, with marked enlargement of the superior orbital fissure. There was a pouch of arachnoid cyst herniated in the orbital fossa. In addition, the infraorbital rim was lower than the contralateral side, allowing inferior displacement of the globe (fig 3).

Her MRI (fig 1) showed the right eyeball has been pushed forward and the orbital contents were pushed medially. There was a temporal lobe arachnoid cyst which had herniated in the orbit. There was also a bony defect at the right posterior parietal region.

We had approached through a fronto-temporal craniotomy on the right side. The bone was thin. Dura was opened and there was an arachnoid cyst at the right temporal pole, which had entered the orbit through the deficient orbital wall. We had excised the arachnoid cyst and also did a cysto-cisternostomy with the basal cistern. The dura attached with the periorbita was very tough and was difficult to dissect. However the volume was reduced by using G-patch $\circledast$. Dura was closed. Then a Titanium mesh was shaped to the floor of the anterior cranial fossa, roof of the orbit and anterior part of the middle cranial fossa (fig 4) and was fixed. The bone flap was also fixed and wound was closed in layers.

Post operatively her recovery was uneventful. Her proptosis had regressed. And her orbital movements had returned. There was some ptosis left due to hypertrophy of the upper eye lid (fig 5). Her stitches were removed on the $8^{\text {th }} \mathrm{POD}$. She was discharged on the $10^{\text {th }}$ POD.

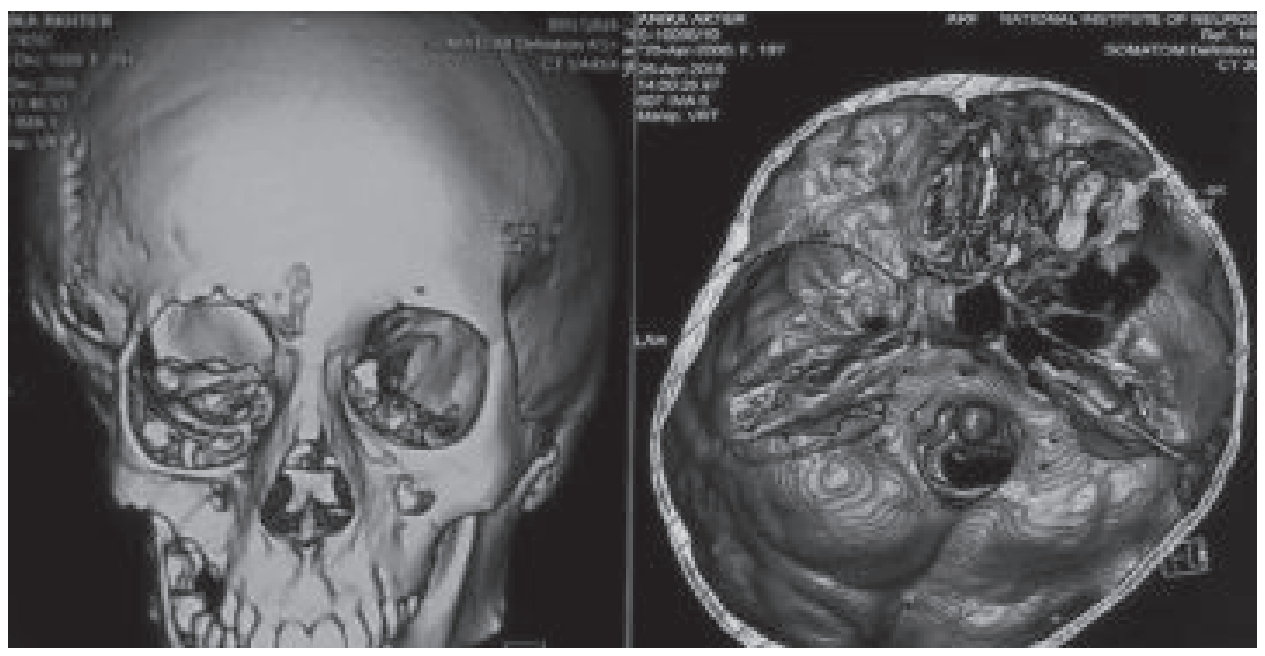

Fig.-3: 3D CT reconstruction of the Patient

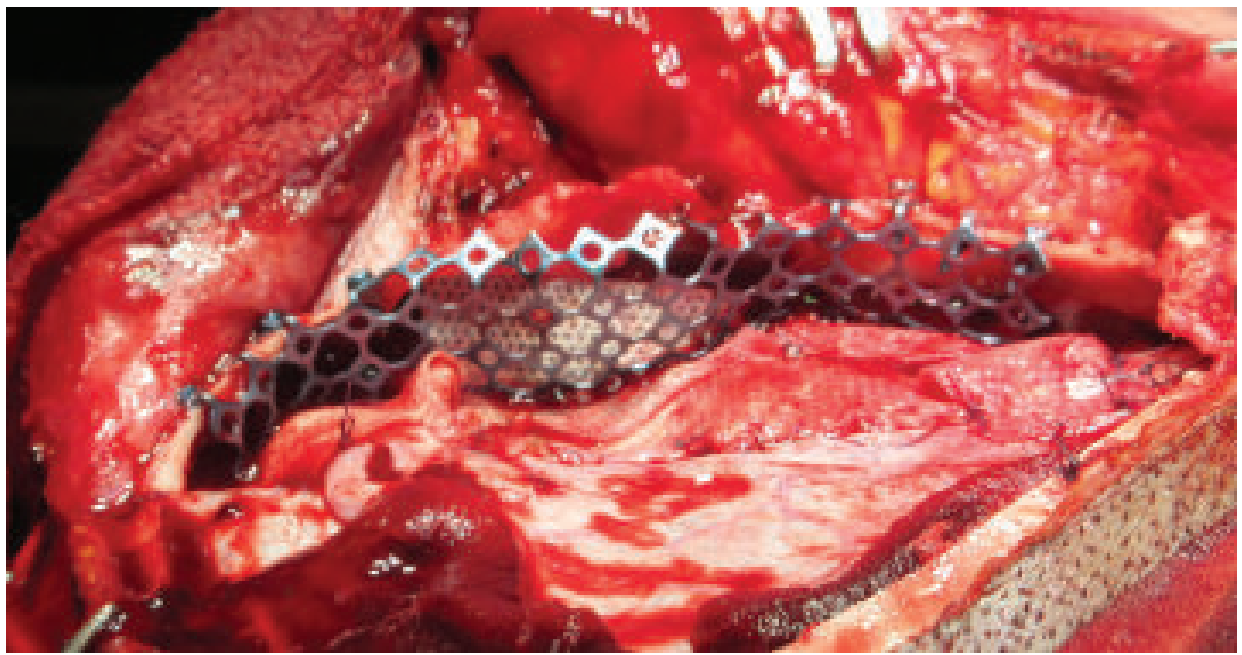

Fig.-4: Intraoperative view reconstruction of Greater wing of the sphenoid with titanium mesh and duroplasty with G-patch. 
On her follow up, her eye movements had improved. But she had proptosis which occurred during crying. Her CT scan (fig 7) showed that there was accumulation of CSF, may due to CSF leaking through G-patch. She was advised for another surgery, which she decided to defer to a later date.

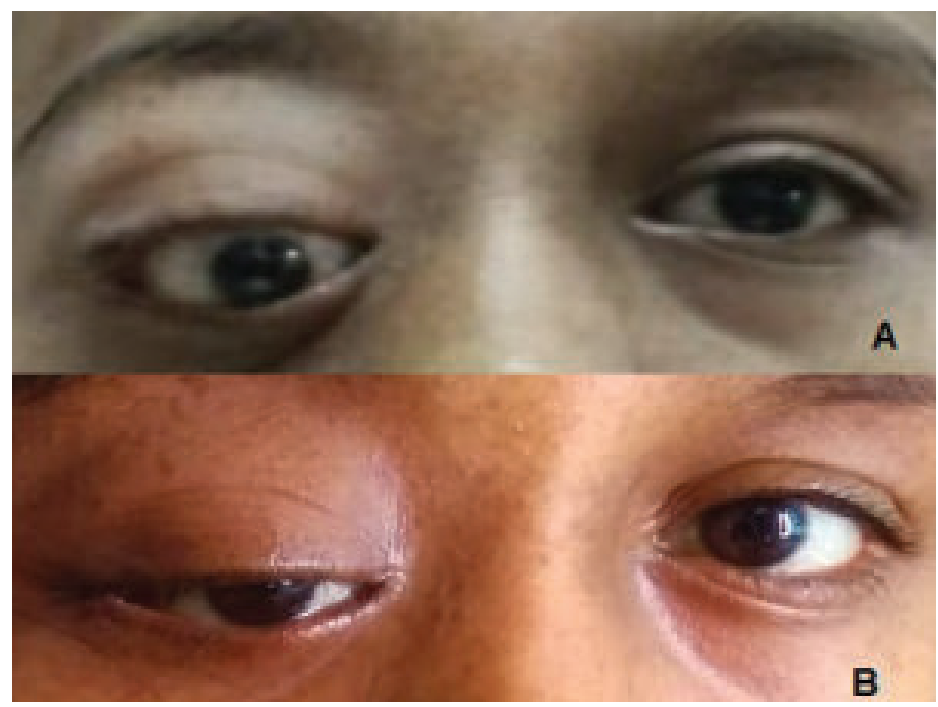

Fig.-5: A. Preoperative view, B.Post operative view. Proptosis has reduced (Reproduced with consent of patient)

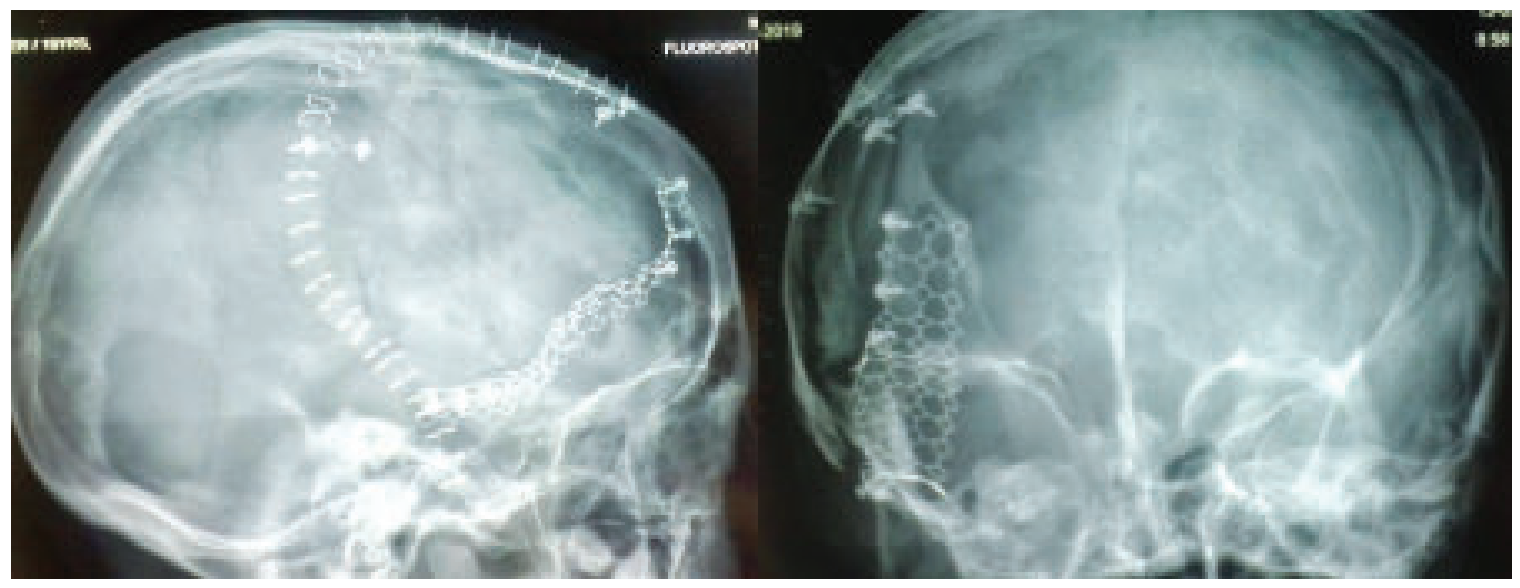

Fig.-6: Post operative X-ray showing reconstructed Cranial base

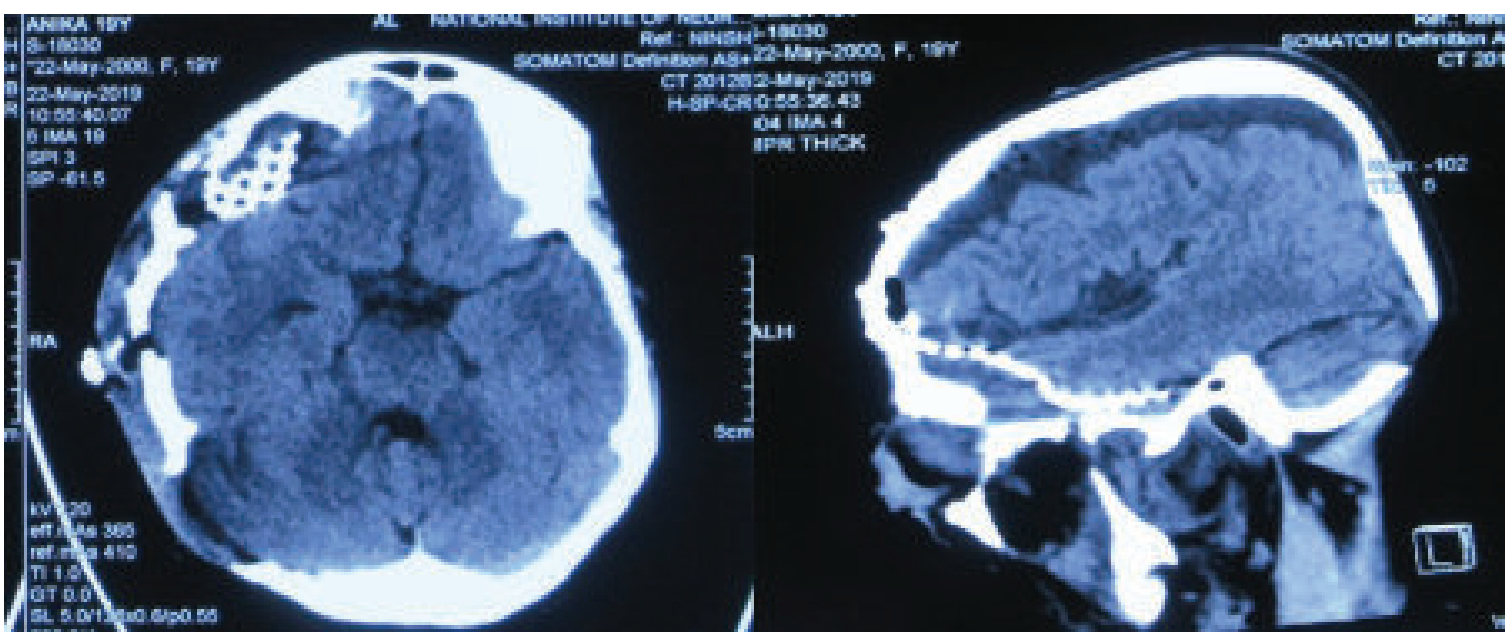

Fig.-7: Post operative CT scan of the patient 


\section{Discussion:}

Use of titanium mesh and vascularized pericranium provides a simple method for reconstruction of large anterior cranial base defects ${ }^{7}$. In one study, the skull base defect was covered with 0.3-mm-thick titanium mesh and hydroxyapatite cement; then, both were covered with a vascularized galeal-pericranial flap ${ }^{8}$. In our patient we did not use bone cement. We had put titanium mesh over the sphenoid bone and we covered it with G-patch.

The most striking skull change was antero-posterior enlargement of the middle cranial fossa with sphenoid bone changes typical of classic sphenoid dysplasia, always with an anterior temporal arachnoid cyst and often with shortening of the orbit ${ }^{9}$. In our patient there was also antero-posterior enlargement of the middle cranial fossa. There was a retrobulbar archnoid cyst which was excised. Then duroplasty was done to close the dura.

Jackson et al classified orbito-temporal neurofibromatosis into three groups(group 1, orbital soft-tissue involvement only with a seeing eye; group 2 , orbital soft-tissue and significant bone involvement with a seeing eye; and group 3, orbital soft-tissue and significant bone involvement with a blind or absent eye). Bone involvement mostly consists of a partial or complete absence of the greater wing of the sphenoid, being an enlargement of the superior orbital fissure with consecutive herniation of the temporal lobe ${ }^{10}$. Therefore, our patient was in group two.

The orbital defect can be corrected in many ways. We chose the intracranial approach as she had a temporal lobe arachnoid cyst also. This had to be resected. Surgical correction can be performed through an intracranial approach but is associated with a high complication rate or when an upper lid incision or coronal flap is used. the lateral orbital approach was suggested as an alternative technique, using an available intraoperative navigation system ${ }^{10}$.

The reconstruction can be done from the cranial side ${ }^{3}$, or from the orbital side with radial titanium mesh ${ }^{2}$. Another process is to use a reverse curved titanium mesh $^{11}$. We have used the transcranial route as it was more convenient for us and placed an extradural titanium mesh. The orbital roof reconstruction to abate the influence of temporal herniation is fundamental ${ }^{6}$.The lateral approach allowed better exposure of the bony defect than a transorbital route, and preservation of the optic nerve ${ }^{12}$. The titanium mesh is malleable and the technique is less time consuming than the autologous bone graft technique. This titanium mesh technique is now used by many surgical teams with better and long-lasting results ${ }^{12}$.

The use of Titanium mesh can provide a solid barrier and separation between the cranium and the orbit or the nasal cavity. It should however be used with local flaps or artificial dura with surgical glue to achieve water tight closure of the dura ${ }^{13}$. We also have used G-patch to cover the titanium mesh and Dura.

\section{Conclusion:}

Sphenoid wing dysplasia results in deformity of the orbital rim. This results in disfigurement and visual disturbance of the patient. By reconstructing the greater wing of sphenoid bone the inferior and anterior displacement of the eye ball can be improved. If the lower rim can be reconstructed at the same time then the cosmetic result would be better. However, the reconstruction brings much comfort and also increases social acceptance of the patient.

\section{References:}

1. Friedrich RE. Reconstruction of the Sphenoid Wing in a Case of Neurofibromatosis Type 1 and Complex Unilateral Orbital Dysplasia with Pulsating Exophthalmos. In Vivo 2011;25(2):287-90.

2. Dale EL, Strait TA, Sargent LA. Orbital Reconstruction for Pulsatile Exophthalmos Secondary to Sphenoid Wing Dysplasia. Annals of Plastic Surgery 2014;72(6):S107-S11.

3. Lotfy M, Xu R, McGirt M, et al. Reconstruction of skull base defects in sphenoid wing dysplasia associated with neurofibromatosis I with titanium mesh. Clinical Neurology and Neurosurgery 2010;112(10):909-14.

4. Cung W, Friedman LA, Khan NE, et al. Cephalometry in adults and children with neurofibromatosis type 1 : implications for the pathogenesis of sphenoid wing dysplasia and the "NF1 facies". European journal of medical genetics 2015;58(11):584-90.

5. Erb MH, Uzcategui $\mathrm{N}$, See RF, et al. Orbitotemporal Neurofibromatosis: Classification and Treatment. Orbit 2007;26(4):223-28.

6. Naran S, Swanson JW, Ligh CA, et al. Sphenoid Dysplasia in Neurofibromatosis: Patterns of Presentation and Outcomes of Treatment. Plastic and Reconstructive Surgery 2018;142(4):518e-26e.

7. Badie B, Preston JK, Hartig GK. Use of titanium mesh for reconstruction of large anterior cranial base defects. Journal of neurosurgery 2000;93(4):711-14.

8. Abe $T$, Anan $M$, Kamida $T$, et al. Surgical technique for anterior skull base reconstruction using hydroxyapatite cement and titanium mesh. Acta neurochirurgica 2009;151(10):1337-38. 
9. Jacquemin C, Bosley TM, Liu D, et al. Reassessment of sphenoid dysplasia associated with neurofibromatosis type 1. American journal of neuroradiology 2002;23(4): 644-48.

10. Friedrich RE, Heiland M, Kehler U, et al. Reconstruction of Sphenoid Wing Dysplasia with Pulsating Exophthalmos in a Case of Neurofibromatosis Type 1 Supported by Intraoperative Navigation Using a New Skull Reference System. Skull Base 2003;13(4):211-16.
11. Concezio DR, Amir S, Gianpiero T, et al. Sphenoid dysplasia in neurofibromatosis type 1: a new technique for repair. Child's Nervous System 2017;33(6):983-86.

12. Niddam J, Bosc R, Suffee TM, et al. Treatment of sphenoid dysplasia with a titanium-reinforced porous polyethylene implant in orbitofrontal neurofibroma: Report of three cases. Journal of Cranio-Maxillofacial Surgery 2014;42(8):1937-41.

13. Taha M, Alnemari H. Skull base reconstruction with titanium mesh for benign complex anterior skull base tumors: case series and review of the literature. J Brain Tumors Neurooncol 2016;1(1):1-5. 